\title{
Mechanical Characterisation of Modified Aramid Fibres Used in Dentistry
}

\author{
A. Malicka-Soczka ${ }^{a}$, L. Domka ${ }^{a, *}$, P. Gajdus ${ }^{b}$ And W. HȩDzelek ${ }^{b}$ \\ ${ }^{a}$ Department of Metalorganic Chemistry, Adam Mickiewicz University \\ Grunwaldzka 6, 60-780 Poznań, Poland \\ ${ }^{b}$ Department of Prosthetic Dentistry, University of Medical Sciences \\ Bukowska 70, 60-812 Poznań, Poland
}

\begin{abstract}
Reconstructive phase of medical treatment, especially in dentistry, requires the use of different polymers. A common laboratory method to increase the fracture resistance of acrylic resin dentures is to include high-resistance synthetic fibres in the processing protocol. However, in the case of aramid fibres the problem of ensuring high durability of bonding between the fibre and acrylic resin has not been solved yet. The aim of the study is to evaluate the mechanical properties and microstructure of acrylic resin reinforced with modified aramid fibres used as fillers or as fibres treated with epoxy resin. In this study mechanical strength of 10 samples of aramid fibres Kevlar (DuPont) and 10 samples of acrylic resin ProBase (Ivoclar) was determined. The controls were unreinforced samples. The three point loading tests were carried out on a universal testing machine Hounsfield. The structures of the fillers and composites were characterised by the scanning electron microscopy. The results are presented graphically in diagrams and table.
\end{abstract}

PACS numbers: 62.20.- $\mathrm{x}, 68.37 . \mathrm{Hk}, 61.41 .+\mathrm{e}$

\section{Introduction}

Reconstructive phase of medical treatment especially in dentistry, requires the use of different polymers. While acrylic resins constitute about $90 \%$ of all materials used in prosthodontics, they are not devoid of drawbacks. These include poor resistance to trauma and abrasion, as well as changes in volume and shape during fabrication process and use. The search for more resistant materials and new technologies to produce removable dentures has led to the development of acrylic resins that have been cross-linked, modified with rubber graft copolymer and also monomer-free materials [1-5]. A common laboratory method to increase the fracture resistance of acrylic resin dentures is based on the inclusion of high-resistance synthetic fibres e.g. glass, carbon, polyethylene or aramid fibres in the processing protocol [6-8]. However, in the case of aramid fibres the problem of durability of bonding between the fibre and the acrylic resin has not been solved yet. As follows from earlier studies, the acrylic samples with aramid fibres undergo delamination $[9,10]$. Therefore, an attempt of novel modifications was made to improve the bonding durability of fibre to the acrylic resin. The aim of the study is to evaluate the mechanical properties and microstructure of acrylic resin reinforced with modified aramid fibres as fillers or as fibres treated with epoxy resin.

\footnotetext{
* corresponding author; e-mail: domkal@amu.edu.pl
}

\section{Material and methods}

Acrylic samples used in the study were made of autopolymerising resin - Pro Base Cold (Ivoclar/ Vivadent, Lichtenstein). The dimensions of the samples were: $65 \mathrm{~mm}$ length, $10 \mathrm{~mm}$ width and $3 \mathrm{~mm}$ thickness. The samples were shaped in a silicon mould. According to the producer's recommendation the polymer to monomer ratio was $15 \mathrm{~g}$ of the powder to $10 \mathrm{ml}$ of the fluid. The fundamental polymerisation was carried out at $40^{\circ} \mathrm{C}$ under pressure of 2 bar for $15 \mathrm{~min}$ in Ivomat (Ivoclar, Lichtenstein). The size of each sample was determined by a calliper and corrected to the recommended dimensions with silica abrasive paper. The aramid fibres Kevlar (Du Pont) packaged by Moratex (Poland) were used for the reinforcement of the acrylic resin.

The first group of samples were made of Kevlar in the form of powder. Fragmented aramid fibres were obtained as a result of grinding the Kevlar fabric and sieving the powder to the mesh size of $0.25 \mathrm{~mm}$. The $10 \%$ or $20 \%$ wt. of powdered Kevlar was added and mixed with the polymer to obtain a homogeneous substance. Preparation of the mixture with the aramid powder did not present any difficulties. Finally, the samples were polymerised. In the acryl samples modified with additional filler the refined aramid fibres were added in the amounts of $10 \%$ or $20 \%$ wt. Kevlar in the form of fine powder was mixed with Pro Base Cold polymer and to this mixture the monomer was added in the amount of $10 \%$ and $20 \%$ wt. in order to fully wet the polymer. The mixture obtained 


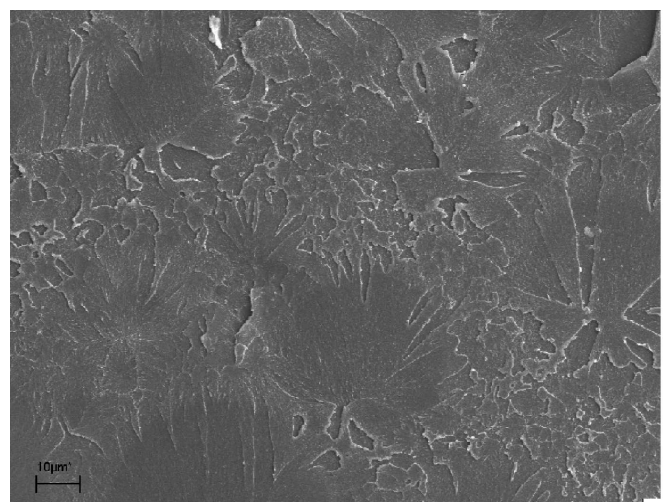

Fig. 1. Pro Base (acrylic control sample) — SEM micrograph.

TABLE

Mechanical strength of the samples studied $[\mathrm{MPa}]$.

\begin{tabular}{c|c|c|c|c|c}
\hline \hline No. & A & B & C & D & E \\
\hline 1 & 62.9 & 109.9 & 96.8 & 83.1 & 87.8 \\
2 & 70.7 & 86.3 & 91.6 & 85.1 & 87.0 \\
3 & 87.6 & 105.9 & 94.1 & 90.3 & 96.8 \\
4 & 96.4 & 110.9 & 93.9 & 100.8 & 89.2 \\
5 & 71.7 & 104.9 & 69.0 & 86.1 & 75.0 \\
6 & 66.4 & 103.0 & 81.0 & 86.1 & 60.8 \\
7 & 81.7 & 100.0 & 72.3 & 54.9 & 74.8 \\
8 & 69.8 & 80.0 & 74.0 & 102.0 & 90.7 \\
9 & 69.6 & 88.8 & 91.4 & 75.7 & 71.0 \\
10 & 61.1 & 98.9 & 75.2 & 89.1 & 81.9 \\
\hline average & 73.8 & 98.9 & 83.9 & 85.3 & 81.5 \\
std. dev. & 11.3 & 10.5 & 10.7 & 13.3 & 10.9 \\
- T 95 & 64.0 & 89.7 & 74.6 & 73.8 & 91.0 \\
+ T 95 & 83.6 & 107.9 & 93.2 & 96.9 & 72.0 \\
\hline A - Pro Base acrylic control sample; \\
B - Pro Base high impact; \\
C - Pro Base with 10\% Kevlar filler; \\
D - Pro Base with 20\% Kevlar filler; \\
E - Pro Base with Kevlar fabric and \\
epoxy resin; \\
std. dev. = standard deviation
\end{tabular}

std. dev. = standard deviation

was placed in the silicon mould and subjected to polymerisation.

The second group consisted of acrylic samples with a piece of Kevlar aramid fabric (thickness $0.30 \mathrm{~mm}$ ). The Kevlar surface was covered with epoxy resin Epidian 624 (Organika, Poland). The epoxy resin was polymerised in two stages, i.e. for $16 \mathrm{~h}$ at room temperature and for $6 \mathrm{~h}$ in a dryer at $80^{\circ} \mathrm{C}$. The acryl samples modified with Kevlar fabric were made with one layer of the fabric covered with epoxy resin of the size: $60 \mathrm{~mm}$ in length,

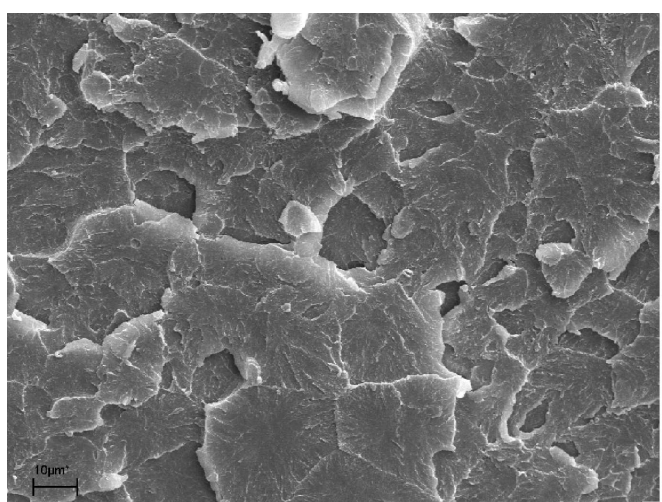

Fig. 2. Pro Base High Impact - SEM micrograph.

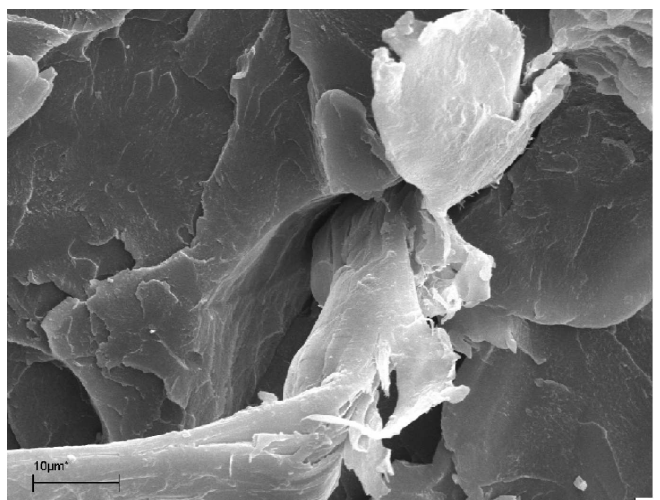

Fig. 3. Pro Base with $10 \%$ Kevlar filler - SEM micrograph.

$8 \mathrm{~mm}$ in width and $0.30 \mathrm{~mm}$ in thickness. The size of the layer ensured the continuity of the plastic on the sample circumference. The appropriate fragment of the Kevlar fabric was inserted into the acryl plastic, pressed into the mould and subjected to polymerization.

The control group were acrylic samples without aramid compounds and the samples of the products from the factory of reinforced acrylic Pro Base High Impact Hot (Ivoclar/Vivadent, Lichtenstein). Ten samples represented each group of the substances studied and they were stored for 4 weeks in water at room temperature before the test. The three-point loading test was carried out on a universal testing machine Hounsfield H5KS. The cross-head speed was $5 \mathrm{~mm} / \mathrm{min}$, support spacing was $50 \mathrm{~mm}$ and the pressure force was $5000 \mathrm{~N}$. Fifty tests of three-point loading were performed (Table). Images of the cross-section of samples representing each group studied were taken at different magnifications, from $250 \times$ to $5000 \times$, on a scanning electron microscope $($ SEM), Zeiss EVO-40, at $19 \mathrm{kV}$ (Figs. 1-5). 


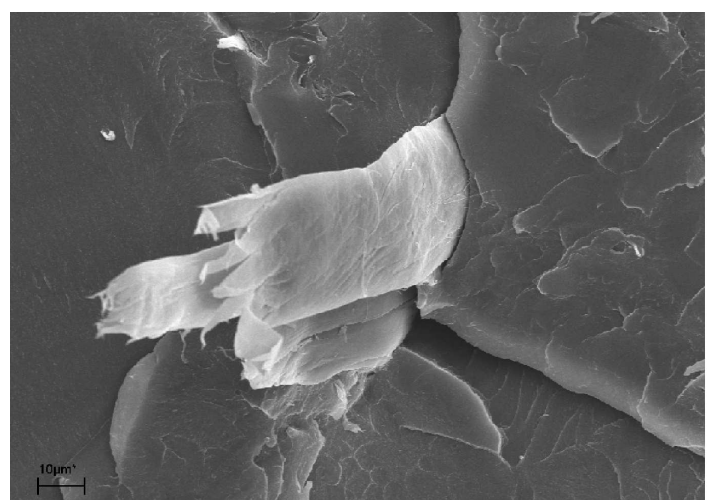

Fig. 4. Pro Base with $20 \%$ Kevlar filler — SEM micrograph.

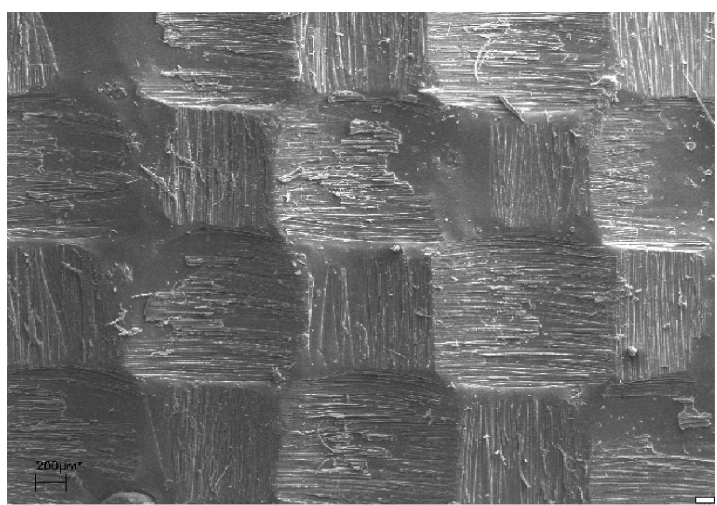

Fig. 5. Pro Base with Kevlar fabric and epoxy resin SEM micrograph.

\section{Results and discussion}

The use of Kevlar fabric in the powdered form or the use of Kevlar fibres covered with epoxy resin as fillers of acryl resin brings an increase in the mechanical strength of the acryl resin material from $73 \mathrm{MPa}$ to $83.9 \mathrm{MPa}$ for the sample with $10 \%$ addition of powdered Kevlar and to $85.3 \mathrm{MPa}$ for the sample with $20 \%$ addition of aramid fibres. The use of Kevlar fibres covered with epoxy resin also increased the mechanical strength but to a lower value of $81.5 \mathrm{MPa}$. The highest mechanical strength of 98.9 MPa was found for the Pro Base High Impact. The differences in the mechanical properties between Pro Base Acrylic Control sample and the Pro Base High Impact sample were statistically insignificant. Modification of the aramid Kevlar fibres by covering them with epoxy resin was intended to stimulate formation of an intermediate layer improving the connection between the Kevlar fibres and acryl resin. Unfortunately, the increase in the mechanical strength of the samples reinforced with pure Kevlar was accompanied by delamination of the acryl resin and aramid fibres.

The SEM images of the Pro Base and Pro Base High Impact samples reveal differences in the surface morphology. The SEM of Pro Base Acrylic Control Sample shows that the surfaces of the fractured ends of this material are smooth, while the surfaces obtained after the fracture of Pro Base High Impact sample show more developed surface. As follows from the SEM image of the surface at the fracture of the sample to which Kevlar was added in the form of ground fibres, the aramid material is regularly distributed and shows good adhesion to acryl resin. The SEM image of the fracture surfaces of the sample of Pro Base with Kevlar fibres covered with epoxy resin (Fig. 5) shows that the fibres are adherent to acryl resin but only in small regions. This effect was macroscopically observed as delamination of the sample at the contact fibre-resin. Despite the modifications, of the sample of Pro Base with Kevlar fabric and Epoxy resin has not shown satisfactory homogeneity and mechanical strength.

\section{Conclusion}

1. The modified aramid fibres applied as fillers have increased the bending strength of the acryl resin models in the degree proportional to the amount of the fibres introduced.

2. The increase in the mechanical strength of the acryl material with Kevlar in the powdered form or in the form of fibres covered with epoxy resin has proved to be statistically insignificant.

3. Because of the hydrophobic character of the aramid Kevlar fibres, it is difficult to obtain good contact at the acryl resin-Kevlar fibre interface.

4. The introduction of Kevlar fabric into the sample studied decreases the bending strength of plates made of acryl resin with this addition.

\section{References}

[1] L. Domka, B. Marciniec, A. Krysztafkiewicz, Method of surface modification of powder materials, Patent PRL 115671 (1983) (in Polish).

[2] A. Krysztafkiewicz, Chem. Stos. 33, 438 (1989) (in Polish).

[3] H. Maciejewski, J. Guliński, B. Marciniec, PolimeryTworzywa Wielkoczasteczkowe 4, 219 (1998) (in Polish).

[4] M.S. Memon, Int. J. Prosthodont. 3, 214 (2001).

[5] P. Pfeiffer, J. Prosth. Dent. 4, 372 (2005).

[6] D.C. Jagger, A. Harrison, K.D. Jandt, J. Oral Rehabil. 26, 185 (1999).

[7] O. Karacaer, A. Dogan, O.M. Dogan, A. Usanmaz, J. Appl. Polym. Sci. 85, 1683 (2002).

[8] S.H. Kim, D.C. Watts, J. Prosthet. Dent. 91, 274 (2004).

[9] P. Gajdus, W. Hȩdzelek, S. Joniak, Prot. Stom. 5, 302 (2003).

[10] C. Bruce, K. Fegely, A. Rajabi-Siahboomi, J. McGinity, Int. J. Pharm. 341, 162 (2007). 